\title{
Aberrant form of Pseudomonas aeruginosa in sputum and cerebrospinal fluid causing infection in a compromised patient
}

\author{
JOHN MIDDLETON AND HERMAN CHMEL \\ From the Infectious Disease Section, Medical Service, Veterans Administration Hospital, East Orange, \\ New Jersey, and the Department of Medicine and Preventive Medicine, College of Medicine and Dentistry \\ of New Jersey, New Jersey Medical School, Newark, New Jersey, USA
}

SUMMARY A patient with non-Hodgkin's lymphoma developed meningitis due to an aberrant form of Pseudomonas aeruginosa observed on Gram stain. The organism was grown on primary isolation media without needing hypertonic media. The significance of aberrant forms in body fluids is discussed.

In-vitro changes in the morphology of many organisms by antibiotics is well documented (Lorian and Atkinson, 1975; Klainer and Perkins, 1972; Lorian and Sabath, 1972). Similarly, the identification and significance of organisms grown or isolated only in hypertonic media (bacterial variants known as L-forms or spheroplasts) was recently reviewed by Louria et al. (1976). We describe a patient in whom organisms atypical in appearance and morphology on initial Gram staining were identified subsequently as Pseudomonas aeruginosa on routine culture media. The clinical significance of this will be discussed.

\section{Case report}

The patient, a 60-year-old man, developed painless cervical adenopathy in September 1975. He had had a right orchidectomy for a seminoma in 1963. Postoperatively he received 3000 rads of irradiation to the para-aortic area without evidence of recurrence. Four months before presentation in 1975 he developed a left foot drop, but did not seek medical advice. On admission biopsy of a left supraclavicular lymph node showed a non-Hodgkin's lymphoma of follicular type. Treatment consisted of 5000 rads of Cobalt-60 to the cervical, supraclavicular, and axillary area bilaterally and the upper mediastinal lymph nodes without complications or significant reduction in lymph node size. A second biopsy of an

Received for publication 26 September 1977 inguinal lymph node was consistent with nonHodgkin's lymphoma.

Chemotherapy was begun with cyclophosphamide, vincristine, and prednisone on 11 January 1976. At this time, the patient began to complain of hoarseness of voice and began to have two to three loose bowel movements a day. Throat examination revealed swelling in the arytenoid area. The loose bowel movements continued and a proctosigmoidoscopy on 16 January showed normal mucosa. Stool examination was unremarkable. On 20 January the patient developed dysphagia. A white blood cell count was $300 / \mathrm{mm}^{3}\left(0.3 \times 10^{9} / 1\right)$ with a haemoglobin of $8.5 \mathrm{~g} / \mathrm{dl}$. Endoscopy on 22 . January revealed three to four petechiae in the submucosa of the stomach. One day later his temperature rose to $103^{\circ} \mathrm{F}$ by mouth. On examination the patient was alert and orientated with a temperature of $103^{\circ} \mathrm{F}$, a blood pressure of $110 / 70 \mathrm{~mm}$ $\mathrm{Hg}$, and was in no acute distress. The neck was supple with negative Kernig's and Brudzinski's signs. The oropharynx was minimally injected without exudate. The lungs had diffuse bilateral rhonchi with no evidence of consolidation. The remainder of the examination was normal. No new lateralising neurological signs or skin lesions were present. Laboratory examination showed a white blood cell count of $300 / \mathrm{mm}^{3}$, haemoglobin $6.4 \mathrm{~g} / \mathrm{dl}$, platelets $27000 / \mathrm{mm}^{3}\left(27 \times 10^{9} / 1\right)$. A lumbar puncture revealed one polymorphonuclear leucocyte but was otherwise unremarkable. Subsequent cerebrospinal fluid blood and urine cultures were negative. Chest 


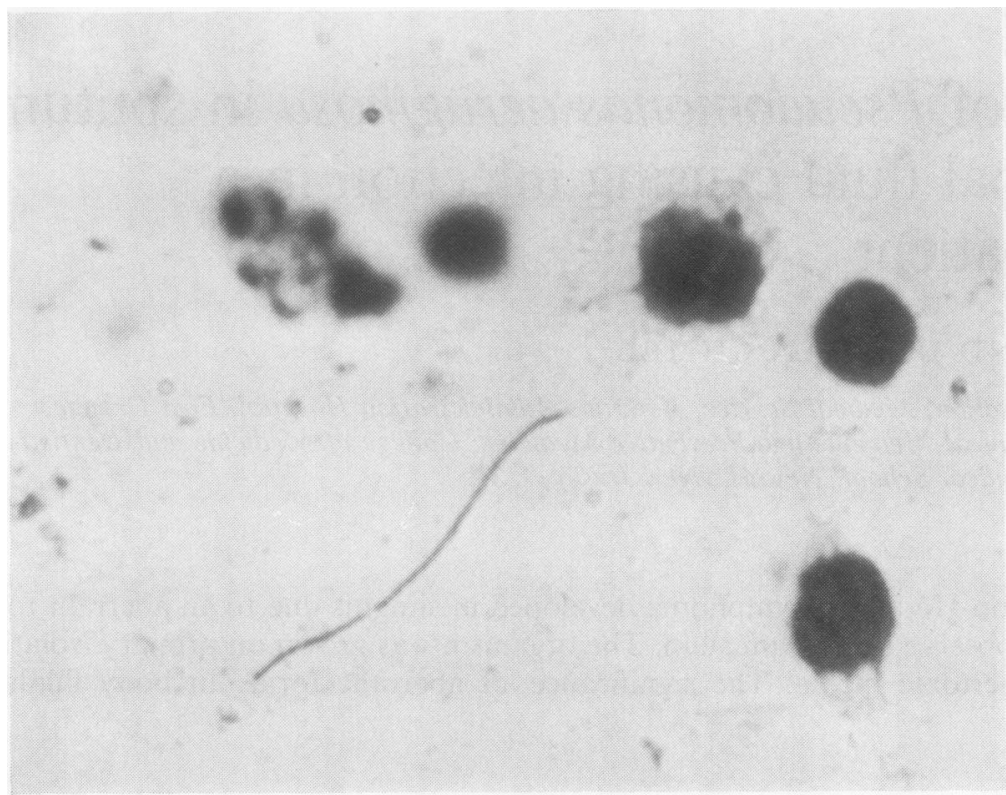

Figure Gram stain of sputum showing long, filamentous Gram-negative rod. $(\times 980)$.

$x$-ray film showed an interstitial pattern in the right lower lung with calcification of the right pleura which was unchanged from September 1975.

The patient was started on cefazolin $500 \mathrm{mg}$ intravenously every six hours plus gentamicin $60 \mathrm{mg}$ intravenously every six hours. His fever continued, rising to $104^{\circ} \mathrm{F}$. Carbenicillin $4.0 \mathrm{~g}$ intravenously every four hours was added. Gram stain of the sputum showed a long, filamentous Gram-negative rod (Figure) which on culture grew only $P s$. aeruginosa. Three to four filamentous Gram-negative rods per high power field were seen. No other organisms were observed. On 27 January an ulcerative lesion developed on the inner aspect of the patient's lower lip. This also subsequently grew $P$ s. aeruginosa. On 29 January the patient became agitated, restless, and incoherent and he had nuchal rigidity with positive Kernig's and Brudzinski's signs. Rales and rhonchi were greatly increased in the right middle lobe area. Chest $x$-ray film showed a new right middle lobe infiltrate. Repeat lumbar puncture showed a sugar of $78 \mathrm{mg} / 100 \mathrm{ml}$, protein $42 \mathrm{mg}$, polymorphonuclear cells $300 / \mathrm{mm}^{3}$ and red blood cells $40 / \mathrm{mm}^{3}(0.04 \times$ $\left.10^{9} / 1\right)$. Gram stain of spinal fluid showed five to six long, filamentous Gram-negative rods per high power field. No other micro-organisms were seen. The stain of the spinal fluid was similar to the Gram stain of the patient's sputum.

The patient died within six hours. Permission for necropsy was not obtained. The cultures of spinal fluid, blood, and sputum were all positive for $P s$. aeruginosa in 24 hours. Interestingly, the $P s$. aeruginosa isolated from the cerebrospinal fluid was resistant by Kirby-Bauer disc diffusion to carbenicillin unlike all other $P$ s. aeruginosa isolated from the patient, which were sensitive to carbenicillin. The organism grew on primary isolation media without the need for hypertonic media. Both the biochemical activities and colonial morphology in primary culture were not unusual and on primary isolation media it had lost its aberrant morphology. The $P s$. aeruginosa was not tested for haemolysin production or serologically tested.

\section{Discussion}

Infections in patients with underlying disease and altered host defences are difficult to treat. Bacteraemias due to Gram-negative organisms, especially $P$ s. aeruginosa, seem to have an affinity for the compromised host (Freid and Vosti, 1968; Young and Armstrong, 1972). McBride et al. (1976) showed that compromised patients are colonised with Gramnegative bacilli, including $P$ s. aeruginosa. However, not all colonised patients become infected with these organisms even when they are present in the patient's immediate environment.

Al-Dujaili and Harris (1975) have looked at the colony morphology, pyocine typing, and haemolysin production of $P s$. aeruginosa in the sputum from hospitalised patients and from their environment to try to discover when Ps. aeruginosa becomes 
'infectious'. The only factor that was closely associated with the ability to produce infection was the in-vitro production of haemolysin. This was not tested in our isolate of Ps. aeruginosa. Other predisposing factors such as abnormal immunoglobulins and neutropenia that may allow the colonising Gramnegative organism to become infectious are operative in the compromised patient (Tapper and Armstrong, 1974).

The terminology of organisms that are atypical in morphology or which require specialised media for growth is confused. Multiple terms, including 'abnormal form', 'aberrant form', 'L-form', and 'spheroplast' are used interchangeably but have specific clinical or microbiological significance for a particular investigator (Lorian and Atkinson, 1975; Louria et al., 1976; Lorian and Waluschka, 1972). The phrase 'aberrant forms' is the most general term and signifies only bacteria that are atypical in appearance but which will grow on routine culture media. Lorian and Atkinson (1975), Lorian and Sabath (1972), and Klainer and Perkins $(1971,1972)$ have excellent illustrations of the in-vitro effects of antibiotics on staphylococcus and Gram-negative organisms, primarily the Enterobacteriacea.

The morphology of bacteria can be altered by a variety of factors, including the culture media used, but the most common cause is the presence of antibiotics. Bacterial appearance can be altered not only by the cell wall-acting antibiotics-for example, penicillins or cephalosporins-but also by agents such as chloramphenicol or nitrofurantoin (Lorian and Atkinson, 1975; Lorian and Sabath, 1972; Klainer and Perkins, 1971, 1972) The mechanism for the production of the abnormal or aberrant forms, which are characteristically elongated, is thought to result from the inhibition of autolytic enzymes that initiate septation (Lorian and Atkinson, 1975). Aberrant forms have been isolated from the urine, cerebrospinal fluid, and in blood cultures (Lorian and Sabath, 1972; Louria et al., 1976; Lorian and Waluschka, 1972; Louria et al., 1969). Our case shows the in-vivo morphological effect of antibiotics on a direct smear from the cerebrospinal fluid and sputum. The appearance was so atypical that the possibility of an anaerobe such as Fusobacterium spp. or Bacteroides spp. was considered.

The aberrant or abnormal forms observed in vivo are in contradistinction to other organisms which require specialised media for growth in the following ways: L-forms are cell-wall deficient and propagate on osmotically stabilising media; protoplasts are usually derived from Gram-positive organisms, lack cell walls, and require appropriate media to grow; spheroplasts are derived from Gram-negative bacteria and usually retain some cell wall but require hypertonic media. Our patient's organism was isolated within 24 hours on routine culture media and did not show the abnormal form on Gram stain in vitro.

In summary, we feel the term 'aberrant' or 'abnormal' forms refer to those organisms that are atypical in morphology on Gram stain; which will grow on routine media; and, as demonstrated by this case, can be clinically significant and initially confusing. 'L-form', 'protoplasts', and 'spheroplasts' should be terms reserved for organisms that require specialised media for growth and identification.

We thank Miss Margaret Wierzbicki and Miss MaryLou Ponzio for secretarial assistance. The work was supported by VA Project No. 1395-01.

\section{References}

Al-Dujaili, A. H., and Harris, D. M. (1975). Pseudomonas aeruginosa infection in hospital: a comparison between 'infective' and 'environmental' strains. Journal of Hygiene, 75, 195-201.

Freid, M. A., and Vosti, K. L. (1968). The importance of underlying disease in patients with gram-negative bacteremia. Archives of Internal Medicine, 121, 418-423.

Klainer, A. S., and Perkins, R. L. (1971). Normal and abnormal morphology of microorganisms: a scanning beam electron microscope study. Journal of the American Medical Association, 215, 1655-1657.

Klainer, A. S., and Perkins, R. L. (1972). Surface manifestations of antibiotic-induced alterations in protein synthesis in bacterial cells. Anti-microbial Agents and Chemotherapy, 1, 164-170.

Lorian, V., and Atkinson, B. (1975). Abnormal forms of bacteria produced by antibiotics. American Journal of Clinical Pathology, 64, 678-688.

Lorian, V., and Sabath, L. D. (1972). Penicillins and cephalosporins: differences in morphologic effects on Proteus mirabilis. Journal of Infectious Diseases, 125, 560-564.

Lorian, V., and Waluschka, A. (1972). Blood cultures showing aberrant forms of bacteria. American Journal of Clinical Pathology, 57, 406-409.

Louria, D. B., Kaminski, T., Grieco, M., and Singer, J. (1969). Aberrant forms of bacteria and fungi in blood or cerebrospinal fluid. Archives of Internal Medicine, 124, 39-48.

Louria, D. B., Kaminski, T., Kapila, R., Tecson, F., and Smith, L. (1976). Study on the usefulness of hypertonic culture media. Journal of Clinical Microbiology, 4, 208-213.

McBride, M. E., Duncan, W. C., Bodey, G. P., and McBride, C. M. (1976). Microbial skin flora of selected cancer patients and hospital personnel. Journal of Clinical Microbiology, 3, 14-20.

Tapper, M. L., and Armstrong, D. (1974). Bacteremia due to Pseudomonas aeruginosa complicating neoplastic 
disease. Journal of Infectious Diseases, 130 (Suppl.), S14-S23.

Young, L. S., and Armstrong, D. (1972). Pseudomonas aeruginosa infections. CRC Critical Reviews in Clinical Laboratory Sciences, 3, 291-347. 